\title{
The antiferromagnetic spin $1 / 2$ chain with competing dimers and plaquettes: Numerical versus exact results
}

\author{
J Richter $\dagger$, N.B.Ivanov $\ddagger$, J.Schulenburg $\dagger$ \\ $\dagger$ Institut für Theoretische Physik, Universität Magdeburg, \\ P.O.Box 4120, D-39016 Magdeburg, Germany \\ $\ddagger$ Institute for Solid State Physics, Sofia, Bulgaria
}

October 22, 2018

\begin{abstract}
We examine the ground state and the excitations of an one-dimensional Heisenberg spin $1 / 2$ antiferromagnet with alternating dimers and four-spin plaquettes (dimerplaquette chain). The properties of the system depend on the competing dimer and plaquette bonds. Several exact, exact-numerical and perturbational results are presented. We find that the system is gapped for all parameter values. The spin pair correlation functions can be characterized by three different correlation lengths for dimer - dimer, dimer - plaquette and plaquette - plaquette correlations. For the latter one we find an effective $S=1$ Haldane like behaviour in the limit of dominating dimer bonds.

Introducing frustration the system undergoes a first order phase transition to a fully dimerized state. Concerning the phase relationships of the ground-state wave function the system represents an example for the exact validity of the Marshall-Peierls sign rule in a strongly frustrated antiferromagnet.

The model considered is related to the recently found 1/5-depleted square-lattice Heisenberg system $\mathrm{CaV}_{4} \mathrm{O}_{9}$.
\end{abstract}

\section{Introduction}

The exciting collective magnetic properties of low-dimensional quantum spin systems have attracted much attention over the last decade. The search for systems with spin-liquid ground states is one subject of continuous interest. Compressible (gapped) and incompressible (gapless) spin liquid phases with more or less exotic ground-state ordering were discussed in particular for the frustrated $J_{1}-J_{2}$ model on the square lattice (see e.g. [i]). The recent discovery of a spin gap in $S=1 / 2$ quasi-two-dimensional $\mathrm{CaV}_{4} \mathrm{O}_{9}$ [2, 3] has stimulated the investigation of quantum disorder and gap formation of systems with different types of antiferromagnetic nearest neighbour (NN) bonds [4, 6, 6, 8, 8, 9, 10]. $\mathrm{CaV}_{4} \mathrm{O}_{9}$ has a layered structure where the magnetic $V^{4+}$ ions have spin $1 / 2$ and form a $1 / 5$ depleted square lattice [11, 12. The minimal model for $\mathrm{CaV}_{4} \mathrm{O}_{9}$ is a $1 / 5$ depleted Heisenberg model, i.e. a model with 4 -spin plaquettes connected at there edges with one neighbouring plaquette. Because of the distortion of the lattice [11, 12] the intra-plaquette $J_{p}$ and the inter-plaquette (dimer) $J_{d}$ bonds might be different. Though in a classical version of this nonfrustrated Heisenberg model the Néel state is the stable ground state for any $J_{p}>0$, $J_{d}>0$, in the quantum case a competition arises between a local singlet formation of a couple of spins along a dimer bond $J_{d}$ and a local singlet formation of the four spins belonging to a plaquette and coupled by $J_{p}$. However, the explanation of the measured spin gap 


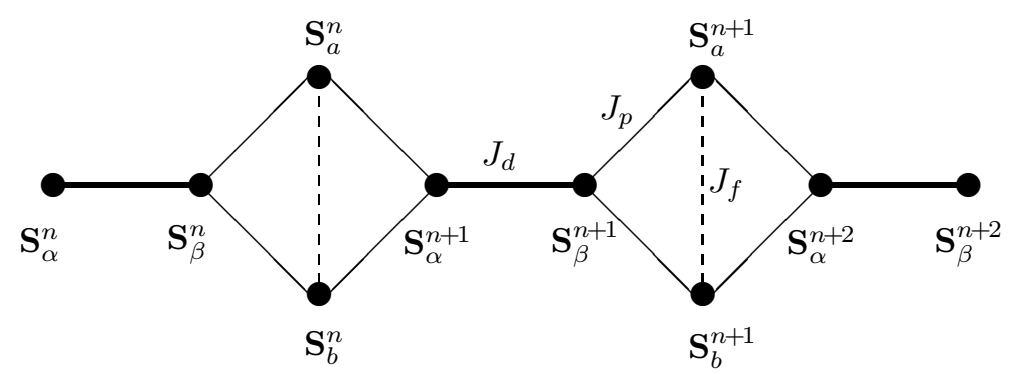

Figure 1: (see text)

by competition between $J_{p}$ and $J_{d}$ would require unreasonable large differences between $J_{p}$ and $J_{d}$. As proposed in several papers 13, 12, 14, 15, 16, 17 one needs additional frustration to get reasonable values for the gap.

In this paper we extend our preliminary discussion 19, 10 of the competition between dimer and plaquette bonds and the role of frustration in the one dimensional counterpart of the depleted square lattice Heisenberg model. This model is simpler than the $2 \mathrm{~d}$ model, but nevertheless it contains non-trivial physics. To our knowledge presently there is no corresponding quasi-1d material but it seems to be possible that it can be synthesised in future.

Though the considered Heisenberg model is a spin $1 / 2$ model it will be shown below that the model contains also elements of the physics of the spin 1 chain which is currently also under intensive discussion (see e.g. [18, 19, 20, 21] and references therein).

The paper is organised as follows: In Section 2 we present the model and elaborate some exact statements concerning eigenvalues and eigenstates of the model. In Section 3 we discuss exact numerical data for chains up to 32 sites as well as analytic results using perturbation theory. Conclusions are given in Section 4.

\section{Model and general results}

The spin 1/2 Heisenberg chain considered here consists of $N$ spins forming $N_{p}=N / 4$ plaquettes which are connected by $N_{d}=N_{p}$ dimer bonds (see Fig.11). The two spins connected by the $n t h$ dimer bond $J_{d}$ we call dimer spins and denote them by $\mathbf{S}_{\alpha}^{n}$ and $\mathbf{S}_{\beta}^{n}$, where the index $\alpha(\beta)$ stands for the left (right) spin. The two spins sitting at the top and the bottom of the $n t h$ plaquette we call plaquette spins and denote them by $\mathbf{S}_{a}^{n}$ and $\mathbf{S}_{b}^{n}$, where the index $a(b)$ stands for the top (bottom) spin. With these notations we write the Hamiltonian

$$
H_{d-p}=J_{d} \sum_{n=1}^{N_{p}} \mathbf{S}_{\alpha}^{n} \mathbf{S}_{\beta}^{n}+J_{p} \sum_{n=1}^{N_{p}}\left(\mathbf{S}_{\beta}^{n} \mathbf{S}_{a}^{n}+\mathbf{S}_{\beta}^{n} \mathbf{S}_{b}^{n}+\mathbf{S}_{a}^{n} \mathbf{S}_{\alpha}^{n+1}+\mathbf{S}_{b}^{n} \mathbf{S}_{\alpha}^{n+1}\right)
$$

$\left(J_{d}, J_{p}>0\right)$. Frustration is introduced by a diagonal antiferromagnetic bond $J_{f}$ connecting a top and bottom plaquette spin (see Fig.1). Then the total Hamiltonian reads

$$
H=H_{d-p}+H_{f}=H_{d-p}+J_{f} \sum_{n=1}^{N_{p}} \mathbf{S}_{a}^{n} \mathbf{S}_{b}^{n} \quad, \quad J_{f} \geq 0 .
$$

For the sake of convenience we consider chains with periodic boundary conditions.

Approximately at the same time when we introduced [9] the above defined dimer-plaquette chain Takano and coworkers [22] considered a so-called diamond chain, built by plaquettes only. The ground state problem of this diamond chain was recently analysed by Niggemann 
et al. [23]. The main difference between both models consists in the existence of the dimer bond in the dimer-plaquette chain. As a consequence, the both models belong to different universality classes. We will briefly discuss some important differences between both models in section 4.

For the above described dimer-plaquette chain (1,:2) we can find the following general statements:

\section{(i) Classical ground state:}

For $J_{f}<J_{p}$ the ground state is a Néel state. The correlations are $\left\langle\mathbf{S}_{a}^{n} \mathbf{S}_{b}^{n}\right\rangle=+S^{2}$ between a bottom and top spin of the same plaquette $\left(J_{f}\right.$ bond), $\left\langle\mathbf{S}_{\alpha}^{n} \mathbf{S}_{\beta}^{n}\right\rangle=-S^{2}$ between two neighbouring dimer spins ( $J_{d}$ bond) and $\left\langle\mathbf{S}_{\beta}^{n} \mathbf{S}_{a(b)}^{n}\right\rangle=\left\langle\mathbf{S}_{\alpha}^{n+1} \mathbf{S}_{a(b)}^{n}\right\rangle=-S^{2}$ between a dimer spin and a neighbouring plaquette spin $\left(J_{p}\right.$ bond). For $J_{f}>J_{p}$ the ground state has twisted plaquette spins. The corresponding correlations of neighbouring spins are $\left\langle\mathbf{S}_{a}^{n} \mathbf{S}_{b}^{n}\right\rangle=$ $S^{2}\left(2 J_{p}^{2} / J_{f}^{2}-1\right),\left\langle\mathbf{S}_{\alpha}^{n} \mathbf{S}_{\beta}^{n}\right\rangle=-S^{2}$ and $\left\langle\mathbf{S}_{\beta}^{n} \mathbf{S}_{a(b)}^{n}\right\rangle=\left\langle\mathbf{S}_{\alpha}^{n+1} \mathbf{S}_{a(b)}^{n}\right\rangle=-S^{2} J_{p} / J_{f}$.

Now we turn to the quantum spin $1 / 2$ case.

\section{(ii) Integrals of motion:}

In addition to the usual integrals of motion (z-component and square of total spin) there are $N_{p}$ local integrals of motion, namely the square of the total spin of the top and bottom spin of a plaquette $n$, i.e

$$
\left[H,\left(\mathbf{S}_{a b}^{n}\right)^{2}\right]_{-}=0 \quad, \quad \mathbf{S}_{a b}^{n}=\mathbf{S}_{a}^{n}+\mathbf{S}_{b}^{n} .
$$

Hence we can classify all eigenstates by the following set of the quantum numbers: energy $E$, z-component of the total spin $M$, square of the total spin $S$, and $N_{p}$ local quantum numbers $S_{p}^{n}$ of $\left(\mathbf{S}_{a b}^{n}\right)^{2}$, where the values for $S_{p}^{n}$ are 0 (singlet) or 1 (triplet). Concerning the correlation function between a top and bottom spin of plaquette $n$ we have $\left\langle\mathbf{S}_{a}^{n} \mathbf{S}_{b}^{n}\right\rangle=-3 / 4$ $(+1 / 4) \quad$ for $\quad S_{p}^{n}=0 \quad\left(S_{p}^{n}=1\right)$.

\section{(iii) Lieb-Mattis theorem and ground state in the nonfrustrated limit:}

In the limit $J_{f}=0$, i.e. $H=H_{p-d}$, the lattice is bipartite and the Lieb-Mattis theorem is valid [24, 25], i.e. the ground state is a singlet $S=0$ of the total spin. As a consequence of the theorem we have $\left\langle\mathbf{S}_{a}^{n} \mathbf{S}_{b}^{n}\right\rangle>0$ since the top and bottom spins of a plaquette $n$ belong to the same sublattice, i.e. the ground state is a singlet of the total spin but all local quantum numbers are $S_{p}^{n}=1\left(n=1, \ldots, N_{p}\right)$.

We notice that the numerical results (see below) indicate that the ground state is a singlet of the total spin for finite frustration, too, which is in accordance with other calculations of the ground state of various frustrated antiferromagnets.

\section{(iv) Mapping onto a spin-1/2-spin-1 chain:}

As a result of (iii) for zero temperature the Hamiltonian (11) can be exactly mapped on a chain with mixed spin $1 / 2$ and spin 1 objects as shown in Fig.2.

This effective model describes not only the ground state, but also all other eigenstates of (11) with $S_{p}^{n}=1$ for all $n=1, \ldots, N_{p}$.

Notice, that antiferromagnetic chains with alternating spins $S=1 / 2$ and $S=1$ where recently discussed as quantum ferrimagnet [26, 27, 28]; however, the effective model here is of different kind since we have twice as much spins $S=1 / 2$ as spins $S=1$. 


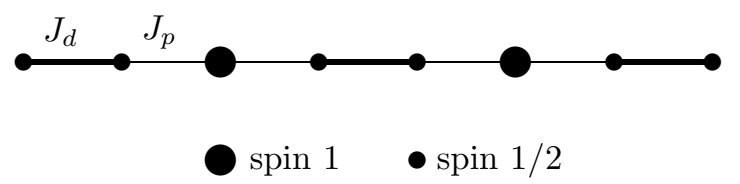

Figure 2: Spin-1/2- spin-1 chain, which is equivalent to the nonfrustrated Hamiltonian (11) for zero temperature.

\section{(v) Product eigenstates:}

We consider now the class of eigenstates which do not correspond to the spin-1/2 spin-1 chain, i.e. we consider states where some of the local quantum numbers $S_{p}^{n}$ are zero. Suppose $S_{p}^{i}=0$ in the plaquette $i$. Then the top and bottom spins of plaquette $i$ form a singlet which is decoupled from all other spins, i.e. we have $\left\langle\mathbf{S}_{a(b)}^{n} \mathbf{S}_{a(b)}^{i}\right\rangle=0(n \neq i)$ and $\left\langle\mathbf{S}_{\alpha(\beta)}^{n} \mathbf{S}_{a(b)}^{i}\right\rangle=0$. Hence the eigenstate can be written in product form

$$
|\Psi\rangle=\left|\left(a_{i}, b_{i}\right)\right\rangle\left|\Psi_{\text {remainder }}\right\rangle
$$

where $\left|\left(a_{i}, b_{i}\right)\right\rangle=\left(\uparrow_{a_{i}} \downarrow_{b_{i}}-\downarrow_{a_{i}} \uparrow_{b_{i}}\right) / \sqrt{2}$ is a pair singlet state of the top and bottom spin of plaquette $i$ and $\left|\Psi_{\text {remainder }}\right\rangle$ is a state describing all the remaining $N-2$ spins forming a corresponding open chain with dimer ends. Suppose $S_{p}^{i}=0$ in $L>1$ plaquettes $i$. Then the eigenstate separates in $L$ pair singlet states of the top and bottom spin of plaquettes $i$ and eigenstates of the finite chain pieces lying between two plaquettes with $S_{p}^{i}=0$. The more plaquettes $i$ in a singlet state $S_{p}^{i}=0$ the shorter the finite chain pieces between two plaquettes with $S_{p}^{i}=0$. The extreme case is the state with $S_{p}^{n}=0$ for all $n=1, \ldots, N_{p}$ plaquettes, where the finite pieces between two plaquettes are just the dimers themselves. This state can be explicitly written as

$$
\left|\Psi_{0, \ldots, 0}\right\rangle=\prod_{n=1}^{N_{p}}\left|\left(a_{n}, b_{n}\right)\right\rangle \prod_{n=1}^{N_{p}}\left|\left(\alpha_{n}, \beta_{n}\right)\right\rangle
$$

where $\left|\left(\alpha_{n}, \beta_{n}\right)\right\rangle=\left(\uparrow \alpha_{n} \downarrow \beta_{n}-\downarrow_{\alpha_{n}} \uparrow_{\beta_{n}}\right) / \sqrt{2}$ is a pair singlet state of a dimer bond $n$. The energy of this state is

$$
E_{0, \ldots, 0}=-\frac{3}{4} J_{d} N_{p}-\frac{3}{4} J_{f} N_{p}
$$

\section{(vi) Eigenstates and energy levels - frustrated versus nonfrustrated model:}

Between the eigenstates and the energy of the nonfrustrated (1) and the frustrated (2) model there exist simple relations due to the fact that $H_{p-d}$ commutes with $H_{f}$. Hence the eigenfunctions of $H_{p-d}$ are not changed including frustration and for the energy contribution of the frustrating part $H_{f}$ only the local quantum numbers $S_{p}^{n}$ are important. Consider any eigenstate of $H_{p-d}$ with energy $E_{p-d}$ and $N_{p}^{s}$ plaquettes with quantum number $S_{p}^{n}=0$ and $N_{p}^{t}$ plaquettes with quantum number $S_{p}^{i}=1\left(N_{p}^{t}+N_{p}^{s}=N_{p}\right)$. Then the energy for the frustrated model $H=H_{p-d}+H_{f}$ is

$$
E_{p-d, f}=E_{p-d}+J_{f}\left(\frac{1}{4} N_{p}^{t}-\frac{3}{4} N_{p}^{s}\right)=E_{p-d}+J_{f}\left(\frac{1}{4} N_{p}-N_{p}^{s}\right)
$$




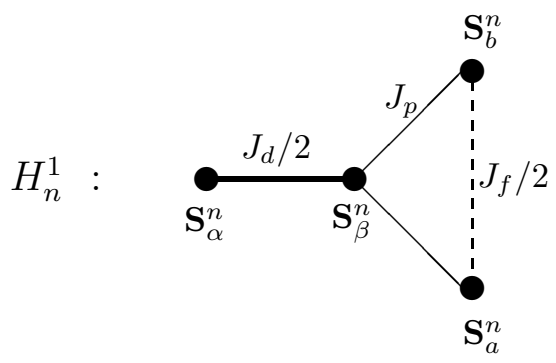

Figure 3: Elementary cluster $H_{n}^{1}$ for the decomposition $H=\sum\left(H_{n}^{1}+H_{n}^{2}\right) \quad$ (see text). The cluster $H_{n}^{2}$ is the mirror immage of $H_{n}^{1}$.

\section{(vii) Upper and lower bound for the critical $J_{f}^{c}$ :}

From Eq. (7) it is obvious that $J_{f}$ favours energetically the singlet formation of plaquette spins and for large $J_{f}$ the singlet product state (5) becomes the ground state of $H$.

According to (iii) the ground state is the lowest eigenstate with $S_{p}^{n}=1$ for all $n=1, \ldots, N_{p}$ for $J_{f}=0$ and has the energy $E_{1, \ldots, 1}^{0}$. Following the ideas of Ref. [23] we used the linear programming scheme to prove that at a critical value $J_{f}^{c}>0$ a first order transition takes place from this ground state directly to the product state (5) of energy $E_{0, \ldots, 0}$ (6) with $S_{p}^{n}=$ 0 for all $n=1, \ldots, N_{p}$. Then the critical $J_{f}^{c}$ is defined by $\left.E_{1, \ldots, 1}^{0}\right|_{J_{f}=J_{f}^{c}}=\left.E_{0, \ldots, 0}\right|_{J_{f}=J_{f}^{c}}$. According to (7) we have $\left.E_{1, \ldots, 1}^{0}\right|_{J_{f}=J_{f}^{c}}=\left.E_{1, \ldots, 1}^{0}\right|_{J_{f}=0}+\frac{1}{4} J_{f}^{c} N_{p} \stackrel{!}{=}-\frac{3}{4}\left(J_{d}+J_{f}^{c}\right) N_{p}$ which yields

$$
J_{f}^{c}=-\frac{3}{4} J_{d}-\left.\frac{1}{N_{p}} E_{1, \ldots, 1}^{0}\right|_{J_{f}=0} .
$$

First we look for an upper bound for $J_{f}^{c}$. We consider $J_{f} \leq J_{f}^{c}$. Then the state with $S_{p}^{n}=1$ for all $n=1, \ldots, N_{p}$ is the ground state and $E_{0, \ldots, 0}(6)$ sets an upper bound for the ground-state energy $E_{1, \ldots, 1}^{0}$.

A lower bound of the ground-state energy is found (see for instance [30]) by a simple cluster decomposition of $H$

$$
\begin{aligned}
H & =\sum_{n=1}^{N_{p}} H_{n}^{1}+H_{n}^{2} \\
H_{n}^{1} & =\frac{J_{d}}{2} \mathbf{S}_{\alpha}^{n} \mathbf{S}_{\beta}^{n}+J_{p} \mathbf{S}_{\beta}^{n}\left(\mathbf{S}_{a}^{n}+\mathbf{S}_{b}^{n}\right)+\frac{J_{f}}{2} \mathbf{S}_{a}^{n} \mathbf{S}_{b}^{n} \\
H_{n}^{2} & =\frac{J_{f}}{2} \mathbf{S}_{a}^{n} \mathbf{S}_{b}^{n}+J_{p} \mathbf{S}_{\alpha}^{n+1}\left(\mathbf{S}_{a}^{n}+\mathbf{S}_{b}^{n}\right)+\frac{J_{d}}{2} \mathbf{S}_{\alpha}^{n+1} \mathbf{S}_{\beta}^{n+1}
\end{aligned}
$$

(see Fig.(3). The lowest energy of $H_{n}^{1}$ and $H_{n}^{2}$ with $S_{p}^{n}=1$ is

$$
E_{n}=-\frac{J_{d}}{8}-\frac{J_{p}}{4}+\frac{J_{f}}{8}-\frac{1}{4} \sqrt{J_{d}^{2}-2 J_{d} J_{p}+9 J_{p}^{2}} .
$$

The lower bound for $E_{1, \ldots, 1}^{0}$ is $2 N_{p} E_{n}$. The resulting inequality $-\frac{3}{4}\left(J_{d}+J_{f}\right) N_{p} \geq E_{1, \ldots, 1}^{0} \geq$ $2 N_{p} E_{n}$ implies the following upper bound for $J_{f}^{c}$

$$
J_{f}^{c} \leq-\frac{J_{d}}{2}+\frac{J_{p}}{2}+\frac{1}{2} \sqrt{J_{d}^{2}-2 J_{d} J_{p}+9 J_{p}^{2}}
$$

Next we look for a lower bound for $J_{f}^{c}$. We use Eq. (8) and replace $E_{1, \ldots, 1}^{0}\left(J_{f}=0\right)$ by a variational energy $E_{v a r}$ of a trial ground state of $H_{p-d}$. Since $E_{v a r} \geq E_{1, \ldots, 1}^{0}\left(J_{f}=0\right)$ the lower bound is

$$
J_{f}^{c} \geq-\frac{3}{4} J_{d}-\frac{1}{N_{p}} E_{v a r} .
$$


For $J_{p}>0$ we are able to find a trial state (see section 3) with $E_{v a r} \leq-N_{p} \frac{3}{4} J_{d}$, i.e. $J_{f}^{c} \geq 0$ is valid for finite $J_{p}$.

\section{(viii) Validity of the Marshall-Peierls sign rule in a frustrated spin system:}

In the limit $J_{f}=0\left(H=H_{p-d}\right)$ the lattice is bipartite and the Marshall-Peierls sign rule is valid [31, 34], i.e. the phase relations of the ground-state wave function are exactly known. Though there are several arguments that these sign rule will survive a finite frustration [32, 33, 34, 35, 36] the validity of the sign rule in nonbipartite frustrated lattice cannot be shown generally.

Based on statements (vi) and (vii) we argue that for all $J_{f}<J_{f}^{c}\left(J_{f}^{c}>0\right)$ the ground state of the nonfrustrated system $H_{p-d}$ remains. Hence the considered plaquette-dimer chain is one example, where the Marshall-Peierls sign rule indeed survives finite frustration.

\section{(ix) Spin gap for large frustration $J_{f}>J_{f}^{c}$ :}

We consider the gap $\Delta$ of the first triplet excitation versus the singlet product ground state $\left|\Psi_{0, \ldots, 0}\right\rangle$ (5). Since in $\left|\Psi_{0, \ldots, 0}\right\rangle$ the top and bottom spins of any plaquette are separated from all other spins, the first triplet excitation is a state $\left|\Psi_{0, \ldots, 0,1,0, \ldots, 0}\right\rangle$ with one triplet for a certain plaquette $i$, i.e. $S_{p}^{i}=1$

$$
\left|\Psi_{0, \ldots, 1,0, \ldots, 0}\right\rangle=\left|\Psi_{-<>-}\right\rangle \prod_{n=1}^{N_{p}-1}\left|\left(a_{n}, b_{n}\right)\right\rangle \prod_{n=1}^{N_{p}-2}\left|\left(\alpha_{n}, \beta_{n}\right)\right\rangle
$$

where in $\prod_{n=1}^{N_{p}-1}$ the plaquette $i$ is excluded and in $\prod_{n=1}^{N_{p}-2}$ the left and right neighbouring dimers of plaquette $i$ are excluded. $\left|\Psi_{-<>-}\right\rangle$represents just the state with $S_{p}^{i}=1$ for the excluded plaquette $i$ and the adjacent dimers $i$ and $i+1$. The degeneracy of the state (13) is $3 N_{p}$.

The excitation gap is the energy difference between the ground state $\left|\Psi_{0, \ldots, 0}\right\rangle$ (5) and $\left|\Psi_{0, \ldots, 0,1,0, \ldots, 0}\right\rangle(13)$

$$
\Delta=E_{1}-E_{0}=\frac{3}{2} J_{d}+J_{f}+E_{-<>-}\left(J_{d}, J_{p}\right),
$$

where $E_{-<>-}\left(J_{d}, J_{p}\right)$ is the energy of the excluded cluster $\diamond$ for $J_{f}=0$. Obviously, $E_{1}-E_{0}$ is independent of size $N$. Since $\left|\Psi_{-<>-}\right\rangle$is a state of only 6 spins there is no problem to calculate $E_{-<>-}\left(J_{d}, J_{p}\right)$, i.e. to find the exact value for $E_{1}-E_{0}$.

\section{Exact diagonalisation versus perturbation theory}

Using Lanczos algorithm we calculate the ground state and several low-lying states for chains with periodic boundary conditions of size $N=8,16,24,32$ (i.e. $N_{p}=2,4,6,8$ plaquettes).

In the limits of $J_{d} / J_{p} \ll 1$ and $J_{p} / J_{d} \ll 1$ we calculate the energies of the singlet ground state and the first triplet excitation by second order perturbation theory. In the limit $J_{d}=0$ the unperturbed ground state is a product of the lowest four-spin plaquette states. In the opposite limit $\left(J_{p}=0\right)$ the ground state of $H_{p-d}$ (1i) is a product of dimer singlets and threefold degenerated triplets of the plaquette spins. The second order perturbation theory in $J_{p}$ leads to the following effective spin Hamiltonian

$$
H_{e f f}=-\frac{3}{4} J_{d} N_{p}-\frac{J_{p}^{2}}{J_{d}} N_{p}+\frac{J_{p}^{2}}{2 J_{d}} \sum_{n=1}^{N_{p}} \mathbf{S}_{a b}^{n} \mathbf{S}_{a b}^{n+1}
$$

where the quantities $\mathbf{S}_{a b}^{n}$ are spin 1 operators, i.e. $\left(\mathbf{S}_{a b}^{n}\right)^{2}=2$. We see that in the limit $J_{p} / J_{d} \ll 1$ the dimer-plaquette $S=1 / 2$ model maps onto the $S=1$ Haldane chain with an effective exchange parameter $J_{\text {eff }}=J_{p}^{2} / 2 J_{d}$. 


\subsection{Groundstate energy, low-lying excitations, spin gap}

First we consider the ground-state energy $E_{0}$. For large frustration $J_{f} \geq J_{f}^{c}$ the explicit expression for $E_{0}$ is given in Eq. (6).

For $J_{f}<J_{f}^{c}$ the ground-state energy obeys Eq. (7) and it is sufficient to consider the unfrustrated Hamiltonian $H_{p-d}$. First we consider the two limits of small dimer exchange $J_{d} \ll J_{p}$ and of small plaquette exchange $J_{p} \ll J_{d}$. In the first case the perturbation theory yields

$$
\frac{E_{0}}{4 N_{p}}=-J_{p}\left[\frac{1}{2}+\frac{1}{4} \frac{43}{576}\left(\frac{J_{d}}{J_{p}}\right)^{2}\right] .
$$

The opposite limit is described by the effective spin 1 model (15), i.e.

$$
\frac{E_{0}}{4 N_{p}}=-\frac{3}{16} J_{d}-\frac{J_{p}^{2}}{4 J_{d}}+\frac{J_{p}^{2}}{8 J_{d}} \epsilon_{H}
$$

where $\epsilon_{H}=-1.401484038971$ [18, 19] is the well-known energy per site of the Haldane chain.

The numerical data for $\mathrm{N}=16$ are drawn in Fig.4. The presented energies belong to the corresponding lowest eigenstate for a given set of local quantum numbers $S_{p}^{n}, n=1, \ldots, 4$. (An exception is the state with total spin $S=1$ and $S_{p}^{n}=1 n=1, \ldots, 4$, which is the first triplet excitation versus the singlet ground state.) The state with highest energy is just the product state $\left|\Psi_{0, \ldots, 0}\right\rangle$ (5), its energy is independent of $J_{p}$ (see Eq. (5)). All presented energies are degenerated for $J_{p}=0$. Increasing $J_{p}$ it follows a quadratic dependence on $J_{p}$ for small $J_{p}$, and a linear dependence on $J_{p}$ for larger $J_{p}$. Notice, that the linear dependence on $J_{p}$ (obtained by perturbation theory in the limit $J_{p} \gg J_{d}$, see (16)) is well established already for $J_{p} \approx J_{d}$.

In all finite systems $(N=8,16,24,32)$ considered in this paper the first triplet excitation has the same local quantum numbers as the ground state, i.e. $S_{p}^{n}=1\left(n=1, \ldots, N_{p}\right)$. The eigenstates with singlets $S_{p}^{n}=0$ for some $n$ have higher energies and and we find that the larger the number of plaquettes with $S_{p}^{n}=0$ the higher the energy.

Next we consider the excitation gap $\Delta$ between the singlet ground state and the first triplet excitation. This triplet excitation is the lowest excitation at all (see Fig.t). The perturbation theory for large $J_{p} / J_{d}$ yields

$$
\Delta=J_{p}\left[1-\frac{1}{3} \frac{J_{d}}{J_{p}}-\frac{61}{576}\left(\frac{J_{d}}{J_{p}}\right)^{2}\right] .
$$

This result was already obtained in 四. In the opposite limit $J_{d} / J_{p} \gg 1$ we can use the results for the Haldane chain 18, 19, and we have

$$
\Delta=\frac{J_{p}^{2}}{2 J_{d}} \Delta_{H}=0.41050 \frac{J_{p}^{2}}{2 J_{d}} .
$$

If we include frustration the situation is not changed for $J_{f}<J_{f}^{c}$ except in in a small parameter region in the vicinity of the transition, i.e for $J_{f} \approx J_{f}^{c}$ the first excitation is not a triplet but a singlet indicating strong frustration effects [37]. For $J_{f}>J_{f}^{c}$ the gap is exactly known in the whole parameter range (see equation (14)).

Numerical data are shown in Fig. 5 , where $\Delta$ is drawn versus $J_{p}$. The linear and quadratic dependences for large and small $J_{p}$ correspond to the perturbation theory (Eqs. (18) and (19). In the scale used for Fig. 5 the data for $N=24$ and $N=32$ almost coincide.

In case of the unfrustrated dimer-plaquette chain with identical NN bonds $J_{p}=J_{d}=1$ the gap is already about $50 \%$ larger then the Haldane gap $\Delta_{H}$, namely $\Delta_{N=24}=0.60922$, $\Delta_{N=32}=0.60906, \Delta_{N \rightarrow \infty}=0.6086$. In accordance with $2 \mathrm{D}$ models for $\mathrm{CaV}_{4} \mathrm{O}_{9}$ the frustration may enlarge the gap, in the considered model we have $\Delta\left(J_{f}+x\right) \geq \Delta\left(J_{f}\right)$ $(x>0)$.

Obviously, though we have a spin $1 / 2$ chain the $\Delta$ is finite for any finite $J_{p}$ which corresponds to the observation that the gapless spectrum of the Bethe chain is an exceptional case. 


\subsection{Critical $J_{f}^{c}$}

The critical point $J_{f}^{c}$ is defined in section 2 , statement (vii). This point coincides with the point of maximal frustration indicated by a maximum in the ground-state energy versus $J_{f}$ precisely at $J_{f}=J_{f}^{c}$.

Upper and lower bounds for $J_{f}^{c}$ are given in Eqs. (11) and (12).

For the estimation of the lower bound we consider a variational state of the form

$$
\left|\Psi_{\text {var }}\right\rangle=\prod_{\substack{n=1 \\ n \text { odd }}}^{N_{p}-1}\left|\uparrow_{a_{n}} \uparrow_{b_{n}}\right\rangle\left|\downarrow_{a_{n+1}} \downarrow_{b_{n+1}}\right\rangle\left|\left\{\alpha_{n}, \beta_{n}\right\}\right\rangle\left|\left\{\beta_{n+1}, \alpha_{n+1}\right\}\right\rangle
$$

where $\left|\uparrow_{a_{n}} \uparrow_{b_{n}}\right\rangle \quad\left(\left|\downarrow a_{n+1} \downarrow_{b_{n+1}}\right\rangle\right)$ is a triplet state of the plaquette spins with z-component $S_{a b}^{n, z}=+1\left(S_{a b}^{n+1, z}=-1\right)$ and $\left|\left\{\alpha_{n}, \beta_{n}\right\}\right\rangle\left|\left\{\beta_{n+1}, \alpha_{n+1}\right\}\right\rangle=$

$\left(1+x^{2}\right)^{-1}\left(\left|\uparrow_{\alpha_{n}} \downarrow_{\beta_{n}}\right\rangle-x\left|\downarrow_{\alpha_{n}} \uparrow_{\beta_{n}}\right\rangle\right)\left(\left|\uparrow_{\beta_{n+1}} \downarrow_{\alpha_{n+1}}\right\rangle-x\left|\downarrow_{\beta_{n+1}} \uparrow_{\alpha_{n+1}}\right\rangle\right)$ is a variational state which interpolates between a dimer singlet state $(x=1)$ and a Néel state $(x=0)$. The calculation of the optimised $x$ is simple, $x=-2 J_{p} / J_{d}+\sqrt{1+4 J_{p}^{2} / J_{d}^{2}}$. The energy of this state $E_{v a r}$ entering Eq. (12) is quite good in the limit of small $J_{p}$ and becomes exact for $J_{p}=0$.

The numerical results are presented in Fig.6. While the lower bound demonstrates that $J_{f}^{c}$ is finite for any finite $J_{p}$ we see the expression (11) for the upper bound is close to the actual value of $J_{f}^{c}$ and can serve as an approximative analytic expression for $J_{f}^{c}$.

\subsection{Pair spin correlation and string order}

At first we consider the limit of large frustrating $J_{f}>J_{f}^{c}$, where the simple product state $\left|\Psi_{0, \ldots, 0}\right\rangle$ (5) is the ground state. Then all spin-spin correlations are zero except $\left\langle\mathbf{S}_{\alpha}^{n} \mathbf{S}_{\beta}^{n}\right\rangle$ and $\left\langle\mathbf{S}_{a}^{n} \mathbf{S}_{b}^{n}\right\rangle$ which take there extreme value $-3 / 4$.

In what follows we discuss the more interesting case $J_{f}<J_{f}^{c}$, i.e. the ground state is that of the unfrustrated $H_{p-d}$. Numerical results for $N=24$ and $N=32$ are shown in Figs. 6.8.9.10. To get a general impression on the distance dependence of the correlations we present in Fig 7 a histogram of the pair correlation versus separation for three values of $J_{p} / J_{d}$. The short range correlations $\left\langle\mathbf{S}_{\alpha}^{n} \mathbf{S}_{\beta}^{n}\right\rangle$ (NN dimer spins), $\left\langle\mathbf{S}_{\beta}^{n} \mathbf{S}_{a(b)}^{n}\right\rangle$ (NN plaquettedimer spins) and $\left\langle\mathbf{S}_{a(b)}^{n} \mathbf{S}_{a(b)}^{n+1}\right\rangle$ (plaquette spins of two neighbouring plaquettes) versus $J_{p} / J_{d}$ are drawn in Fig. 8 and the spin correlations for large separations, namely $\left\langle\mathbf{S}_{a(b)}^{n} \mathbf{S}_{\beta}^{n+3}\right\rangle$ (dimer spin - plaquette spin), $\left\langle\mathbf{S}_{a(b)}^{n} \mathbf{S}_{a(b)}^{n+3}\right\rangle$ (plaquette spin - plaquette spin) and $\left\langle\mathbf{S}_{\alpha}^{n} \mathbf{S}_{\beta}^{n+3}\right\rangle$ (dimer spin - dimer spin) are given in Fig.9.

In the dimer limit $\left(J_{p} \ll J_{d}\right)$ the dimer and plaquette spins are decoupled, i.e. $\left\langle\mathbf{S}_{\alpha(\beta)}^{n} \mathbf{S}_{a(b)}^{m}\right\rangle=$ 0 . Otherwise, the NN dimer correlation $\left\langle\mathbf{S}_{\alpha}^{n} \mathbf{S}_{\beta}^{n}\right\rangle$ takes its extreme value $-3 / 4$ while for spins belonging to different dimers $\left\langle\mathbf{S}_{\alpha(\beta)}^{n} \mathbf{S}_{\alpha(\beta)}^{m}\right\rangle(n \neq m)$ goes to zero, too. However, though the dimer and plaquette spins and the non-neighbouring dimer spins are not correlated there is a well-pronounced correlation between more distant plaquette spins with several dimer spins in between. This is a typical quantum effect; a classical spin chain with NN exchange would be split in separated pieces at that point where the NN correlations are zero. The correlation between spins of different plaquettes is described by the effective Haldane chain (15), the numerical results indicate that this effective Hamiltonian describes the chain well until $J_{p} \approx 0.1 \ldots 0.15 J_{d}$. For example for $J_{p}=0.1 J_{d}$ the plaquette-plaquette correlation $\left\langle\mathbf{S}_{a(b)}^{n} \mathbf{S}_{a(b)}^{m}\right\rangle$ differs from the corresponding Haldane correlation $\frac{1}{4}\left\langle\mathbf{S}_{n} \mathbf{S}_{m}\right\rangle$ (indicated by crosses + in Fig.7) by less then $3 \%$.

In the plaquette limit $\left(J_{p} \gg J_{d}\right)$ the ground state becomes a simple product state of the lowest four-spin plaquette states. Hence, for $J_{p} \rightarrow \infty$ we have $\left\langle\mathbf{S}_{\alpha}^{n} \mathbf{S}_{\beta}^{m}\right\rangle \rightarrow 0,\left\langle\mathbf{S}_{a(b)}^{n} \mathbf{S}_{a(b)}^{l}\right\rangle \rightarrow$ $0(n \neq l)$ and $\left\langle\mathbf{S}_{\beta}^{n} \mathbf{S}_{a(b)}^{n}\right\rangle \rightarrow-0.5$. Already for $J_{p}=J_{d}$ the pair correlation drops down very rapidly (cf. Fig. (]) and besides the correlation along the $J_{p}$ bond, $\left\langle\mathbf{S}_{\beta}^{n} \mathbf{S}_{a(b)}^{n}\right\rangle$, only extremely short-ranged correlations are present. 
There is comparably small region around $J_{p} \sim 0.3 \ldots 0.4 J_{d}$ where we have a balance between $J_{p}$ and $J_{d}$ and all correlations are well-pronounced. Since we have a gap for all $J_{p}>0$ we argue that all correlations show exponential decay but with different correlation lengths $\xi_{d d}$ for the dimer-dimer, $\xi_{d p}$ for the dimer-plaquette and $\xi_{p p}$ for the plaquetteplaquette correlations. The obtained results suggest that $\xi_{p p}$ is is quite large for $J_{p} / J_{d} \ll 1$ $\left(\xi_{p p}=\xi_{\text {Haldane }} \approx 6.03\right.$ 18, 19, 21] for $\left.J_{p} / J_{d} \rightarrow 0\right)$, With increasing $J_{p}$ there is a continuous decrease of $\xi_{p p}$ up to $\xi_{p p} \rightarrow 0$ for $J_{p} \rightarrow \infty$. Otherwise, $\xi_{d d}$ and $\xi_{p d}$ are extremely small for $J_{p} / J_{d} \ll 1$ and $J_{p} / J_{d} \gg 1$ but show a maximum for $J_{p} / J_{d} \sim 0.35\left(\xi_{d d}\right)$ and $J_{p} / J_{d} \sim 0.3$ $\left(\xi_{p d}\right)$.

Finally we discuss the string order parameter describing possible hidden order in spin 1 chains [18, 19, 20, 21]. This order parameter is defined as

$\mathcal{O}_{\pi}^{z}(i, j)=\left\langle S_{i}^{z}\left(\exp \sum_{k=i+1}^{j} i \pi S_{k}^{z}\right) S_{j}^{z}\right\rangle$ where the $S_{i}^{z}$ are spin one objects. For the Haldane spin 1 chain we have $\mathcal{O}_{\pi}^{z}=\lim _{|i-j| \rightarrow \infty} \mathcal{O}_{\pi}^{z}(i, j)=0.374325096$ and the value $\mathcal{O}_{\pi}^{z}(1,4)$ for the third neighbour is already close to $\mathcal{O}_{\pi}^{z}$ 18. For the considered dimer plaquette chain we write

$$
\mathcal{O}_{\pi}^{z}(n, m)=\left\langle S_{a b}^{n, z}\left(\exp \sum_{k=n+1}^{m} i \pi S_{a b}^{k, z}\right) S_{a b}^{m, z}\right\rangle
$$

with $S_{a b}^{m, z}$ defined in (3). The results are shown in Fig.10. In agrrement with pair correlation we observe a Haldane like behaviour until $J_{p} \approx 0.1 \ldots 0.15 J_{d}$ which is followed by region $0.15 J_{d} \lesssim J_{p} \lesssim 0.6 J_{d}$ where a crossover from the Haldane behaviour to the product state with vanishing pair correlations and vanishing string order takes place.

\section{Conclusions}

We have calculated the ground-state properties and low-lying excitations for a $S=1 / 2$ chain with alternating dimers and plaquettes (see Eqs. (11) and (2) and Fig.11). This model is in some sense the $1 \mathrm{D}$ counterpart of the $1 / 5$ depleted square lattice Heisenberg model for $\mathrm{CaV}_{4} \mathrm{O}_{9}$.

While the classical ground state of unfrustrated model $H_{p-d}$ is the Néel state there is a quantum competition between local singlet formation on the dimers or on plaquettes for $S=1 / 2$. Besides of exact diagonalisation and perturbation theory results we have given several general and rigorous statements.

The main results can be summarized as follows. The ground-state properties and a class of excitations of $H_{p-d}$ can be mapped on a mixed spin-1/2-spin-1 chain with two dimer $S=1 / 2$ spins and one effective $S=1$ plaquette spin in the unit cell. In the limit of small plaquette bonds $J_{p} \ll J_{d}$ the ground-state correlations of the effective $S=1$ plaquette spins can be described by a Haldane chain. Increasing the ratio $J_{p} / J_{d}$ a crossover takes place from the effective Haldane chain to a ground state described by a product of plaquette singlet states. The pair correlations are characterized by three different correlation lengths for dimer-dimer, dimer-plaquette and plaquette-plaquette correlations. In the limit $J_{p} \ll J_{d}$ the correlations between plaquette and dimer spins as well as between non-neighbouring dimer spins vanish, but surprisingly the correlation between plaquette spins are well pronounced. (Note that this is a purely quantum effect and has no classical analogue). In the opposite limit $J_{p} \gg J_{d}$ all correlation lengths are extremly short ranged. Though the dimer-plaquette chain $H_{p-d}$ is a $S=1 / 2$ model the first triplet excitation is separated by a gap for all parameter values except $J_{p}=0$. This is consistent with the observation that the gapless ground state of the Bethe chain is quite unstable against the addition of relevant operators to create a gap in the excitation spectrum (see for instance the $S=1 / 2$ chain with alternating NN bonds [38]).

Frustration can be introduced in the model in a simple way by adding an antiferromagnetic interaction of strength $J_{f}$ between the top and the bottom spin of a plaquette (see (2) and Fig.1). In the frustrated model we find a first order quantum phase transition at a finite critical frustration $J_{f}^{c}$ between the ground-state phase described above and a completely 
dimerized phase, which is similar to a recently described first-order transition in antiferromagnetic $S=1 / 2$ coupled chains [39, 23]. Close to the transition the first excitation over the ground state is not a triplet but a singlet, which is a signature of strong frustration [37. The considered model is one example for the rigorous validity of the Marshall-Peierls sign rule in a frustrated antiferromagnet.

As mentioned in section 2 we will briefly point out some important differences between the dimer-plaquette chain discussed in this paper and the spin 1/2 diamond chain considered in [22, 23. In the limit of small frustration the diamond chain corresponds to a quantum ferrimagnet. The ground state of this ferrimagnet has macroscopic total spin $S=N / 6$, is long-range ordered and the spectrum is gapless [26, 27, 28]. On the other hand, the dimer-plaquette chain has a singlet ground state without long-range order and has a gap for all parameter values considered here. However, the common property of both models consists in the product singlet state for large frustration.

Finally we mention that a straightforward extension of the model is obtained by adding further plaquette spins $\mathbf{S}_{c}^{n}, \mathbf{S}_{d}^{n}, \ldots$ The ground-state properties of this extended model could be mapped on a corresponding mixed spin-1/2-spin- $p / 2$ chain ( $p$ is the number of spins in a plaquette $n$ ) with two dimer $S=1 / 2$ spins and one effective $S=p / 2$ plaquette spin in the unit cell.

\section{Acknowledgments}

This work was supported by the DFG (Ri 615/1-2) and Bulgarian Science foundation, Grant F412/94. The authors are indebted to U.Schollwöck for fruitful discussions and to P.Tomczak for reading the manuscript.

\section{References}

[1] F.Figuereido, A.Karlhede, S.Kivelson, S.Sondhi, M.Rocek, D.S.Rokhsar, Phys.Rev. B 41, 4619 (1990); J.Richter, Phys. Rev. B 47, 5794 (1993); J.Oitmaa and Z.Weihong, Phys. Rev. B 54, 3022 (1996).

[2] S. Taniguchi, T. Nishikawa, Y. Yasui, Y. Kobayashi, M. Sato, T. Nishioka, M. Kontani, and K. Sano, J. Phys. Soc. Jpn. 64, 2758 (1995).

[3] T.Ohama, H.Yasuoka, M.Isobe, and Y.Ueda, J. Phys. Soc. Jpn. 66, 23 (1997).

[4] N. Katoh and M. Imada, J. Phys. Soc. Jpn. 64, 4105 (1995).

[5] N.B.Ivanov, S.E.Krüger and J.Richter, Phys.Rev.B 53, 2633 (1996).

[6] M. Albrecht and F. Mila, Phys. Rev. B 53, R2945 (1996).

[7] M.Troyer, H. Kontani, K.Ueda, Phys. Rev. Lett. 76, 3822 (1996).

[8] S.V. Meshkov and D.Förster, J.Phys.:Condens.Matter 8, 7917 (1996).

[9] J. Richter and N. B. Ivanov, Proceedings of the LT21, Prague August 1996 in Czech. J. Phys. 46, 1919 (1996).

[10] N.B.Ivanov and J.Richter, Phys. Lett. A 232, 308 (1997).

[11] J.-C. Bouloux and J.Galy, Acta Crystallographica B 20, (1973) 1335.

[12] O.A.Starykh, M.E.Zhitomirsky, D.I.Khomski, R.R.P.Singh and K.Ueda, Phys. Rev. Lett. 77, 2558 (1996).

[13] K. Ueda, H. Kontani, M. Sigrist, and P. A. Lee, Phys. Rev. Lett. 76, 1932 (1996). 
[14] M.P.Gelfand, Z.Weihong, R.R.P.Singh, J.Oitmaa and C.J.Hamer, Phys. Rev. Lett. 77, 2794 (1996).

[15] S.R.White, Phys. Rev. Lett. 77, 3633 (1996).

[16] S.Sachdev and N.Read, Phys. Rev. Lett. 77, 4801 (1996).

[17] I.Bose and A.Gosh, preprint cond-mat 9705162

[18] S.R.White and D.A.Huse, Phys.Rev.B 48, 3844 (1993).

[19] O.Golinelli, Th. Jolicœur, and R.Lacaze, Phys.Rev.B 50, 3037 (1994).

[20] H.-J.Mikeska, Chaos, Solitons, Fractals 5, 2585 (1995).

[21] A.Kolezhuk, R.Roth, and U.Schollwöck, Phys.Rev.B 55, 8928 (1997).

[22] K.Takano, K.Kubo and H.Sakamoto, J. Phys.:Condens.Matter 8, 6405 (1996).

[23] H.Niggeman, G.Uimin and J.Zittartz, J. Phys.:Condens.Matter 9, 9031 (1997).

[24] E. H. Lieb and D. C. Mattis, J. Math. Phys. 3, 749 (1962).

[25] D. J. Klein, J. Chem. Phys. 77, 3098 (1982).

[26] S.Brehmer, H.-J. Mikeska and S.Ymamoto J. Phys.:Condens.Matter 9, 3921 (1997).

[27] S.K.Pati, S.Ramasesha and D.Sen J. Phys.:Condens.Matter 9, 8707 (1997).

[28] N.B.Ivanov preprint cond-mat 9712014.

[29] Y.Xiang, Phys.Rev.B 52, 12485 (1995).

[30] R.Valenti, J.Stolze, and P.J.Hirschfeld, Phys.Rev.B 43, 13743 (1991).

[31] W.Marshall, Proc.Roy.Soc. A232, 48 (1955).

[32] H.Kitatani, in Computational Approaches in Condensed- Matter Physics, eds. S. Miyashita, M.Imada and H.Takayama, Springer, 1992, p.181.

[33] K. Retzlaff, J.Richter, and N.B.Ivanov, Z.Phys.B 93, 21 (1993).

[34] J. Richter, N. B. Ivanov, and K. Retzlaff, Europhys. Lett. 25, 545 (1994).

[35] C.Zeng and J.B.Parkinson, Phys.Rev.B 51, 11609 (1995); R.Bursill, G.A.Gehring, D.J.J.Farnell, J.B.Parkinson, T.Xiang and C.Zeng, J.Phys.:Condens.Matter 7, 8605 (1995).

[36] A.Voigt, J. Richter, and N.B. Ivanov, Physica A, in press.

[37] B.Bernu, P.Lecheminant, C.Lhuillier and L.Pierre, Phys.Scr. 49, 192 (1993); B.Bernu, P.Lecheminant, C.Lhuillier, L.Pierre, P.Sindzingre, C.Waldtmann and H.-U.Everts, preprint 1997; P.Lecheminant, B.Bernu, C.Lhuillier, L.Pierre, and P.Sindzingre preprint cond-mat 9706167 (1997).

[38] M.C.Gross and D.S.Fisher, Phys.Rev.B 19, 402 (1979); I.Affleck, D.Gepner, H.J.Schulz and T.Ziman, J.Phys.A 22, 511 (1989).

[39] Y.Xiang, Phys.Rev.B 52, 12485 (1995). 
Figure 4: Energy eigenvalues versus $J_{p} / J_{d}$ for the unfrustrated model $H_{p-d}$ with $N=16$ sites. The four numbers in brackets give the local quantum numbers $S_{p}^{n}, n=1, \ldots, 4 ; S$ is the quantum number of the total spin.

Figure 5: Excitation gap between the singlet ground state and the lowest triplet excitation. The dashed lines and the squares correspond to the unfrustrated case. The solid line corresponds to the frustrated case with $J_{f}>J_{f}^{c}$, where the gap is independent of $N$. For $J_{f}<J_{f}^{c}$ tha gap of the frustrated and the unfrustrated model coincide. The critical point for $N=24$ is at $J_{p}=0.534 J_{d}$.

Figure 6: Critical frustration $J_{f}^{c}$ versus $J_{p}$ for $\mathrm{N}=24$ (crosses) and $\mathrm{N}=16$ (solid line) and upper and lower bounds (see text). Above the critical line the ground state of $H$ is the the fully dimerized product state (5) and below the critical line the ground state of the total Hamiltonian $H$ coincides with that one of $H_{p-d}$ (Eq. (11)).

Figure 7: Spin pair correlations $\langle\mathbf{S}(0) \mathbf{S}(j)\rangle$ versus separation of the unfrustrated chain $H_{p-d}$ of length $N=32$ sites for three values of $J_{p} / J_{d}$. For the spin-spin separation the number of NN steps from the reference spin at 0 to spin at $j$ is taken. Left $(\mathrm{P})$ side of the Figures - reference spin $\mathbf{S}(0)$ is a plaquette spin $\mathbf{S}_{a(b)}^{n}$, i.e. the correlations $j=1,2,4,5,7,8,10,11$ are plaquette-dimer correlations, the correlations $j=3,6,9,12$ are plaquette-plaquette correlations (cf. Fig.1). Right (D) side of the Figures - reference spin $\mathbf{S}(0)$ is a dimer spin $\mathbf{S}_{\alpha}^{n}$, i.e. the correlations $j=1,3,4,6,7,9,10,12$ are dimer-dimer correlations, the correlations $j=2,5,8,11$ are dimer-plaquette correlations (cf. Fig.11). The crosses indicate the correlations of the corresponding Haldane chain of length $N=8$.

Figure 8: Short range spin correlations versus $J_{p}$ for the unfrustrated chain $H_{p-d}$ and $N=24$ sites. $\left\langle\mathbf{S}_{\beta}^{n} \mathbf{S}_{a(b)}^{n}\right\rangle-\mathrm{NN}$ dimer spin-plaquette spin, $\left\langle\mathbf{S}_{a}^{n} \mathbf{S}_{a}^{n+1}\right\rangle$ - plaquette spin plaquette spin of neighbouring plaquettes, $\left\langle\mathbf{S}_{\alpha}^{n} \mathbf{S}_{\beta}^{n}\right\rangle$ - NN dimer spin - dimer spin

Figure 9: Spin correlations for largest spin separations versus $J_{p}$ for the unfrustrated chain $H_{p-d}$ and $N=24 .\left\langle\mathbf{S}_{a}^{n} \mathbf{S}_{\beta}^{n+3}\right\rangle$ - dimer spin - plaquette spin, $\left\langle\mathbf{S}_{a}^{n} \mathbf{S}_{a}^{n+3}\right\rangle$ - plaquette spin - plaquette spin, $\left\langle\mathbf{S}_{\alpha}^{n} \mathbf{S}_{\beta}^{n+3}\right\rangle$ - dimer spin - dimer spin

Figure 10: String order $\mathcal{O}_{\pi}^{z}(n, m)$ (see Eq. (21) for $(n, m)=(1,2),(1,3),(1,4)$ versus $J_{p} / J_{d}$ for the unfrustrated chain $H_{p-d}$ of length $N=24$ and $N=32$. 


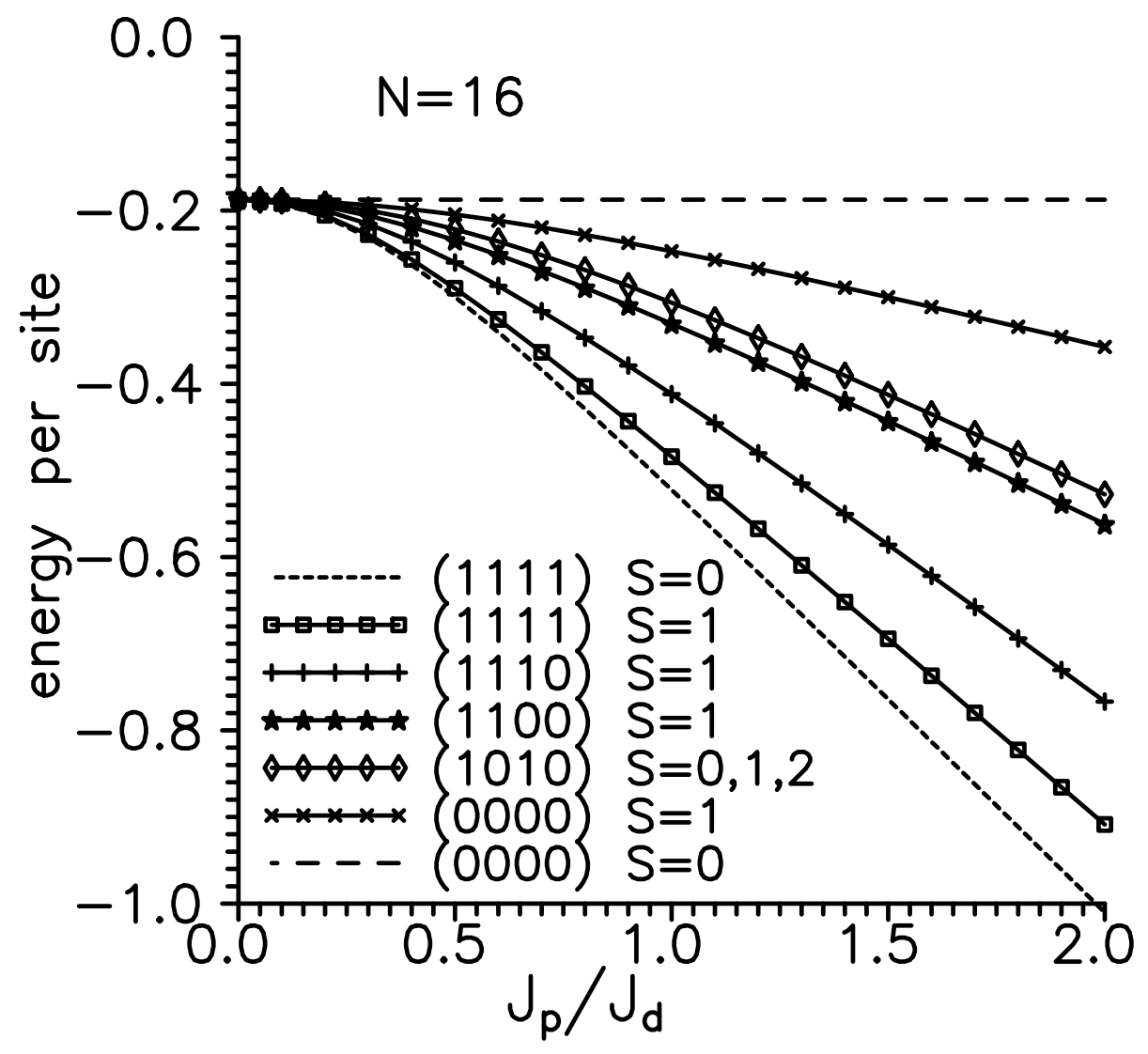

Fig.4 J.Richter et. al. 


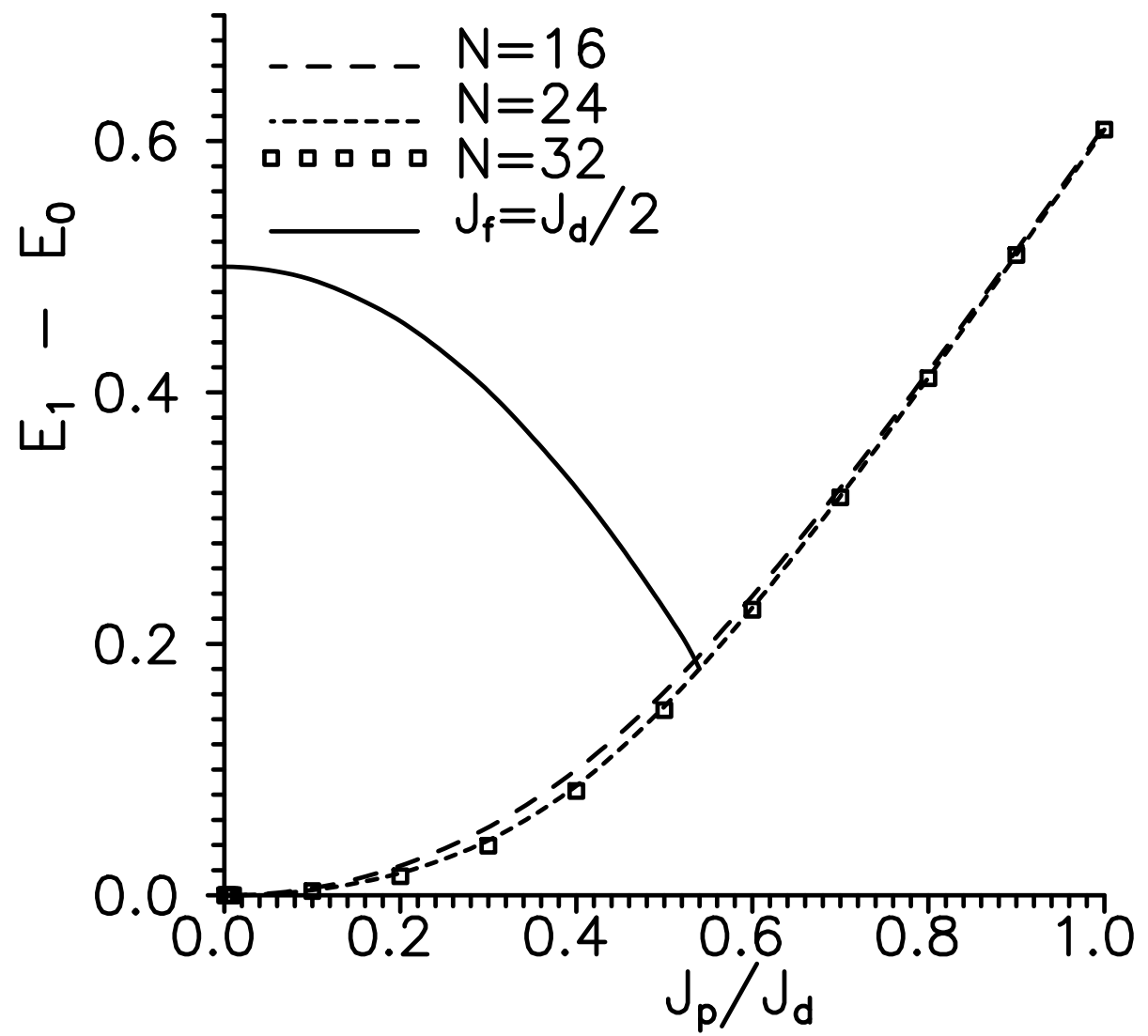

Fig.5 J.Richter et. al. 


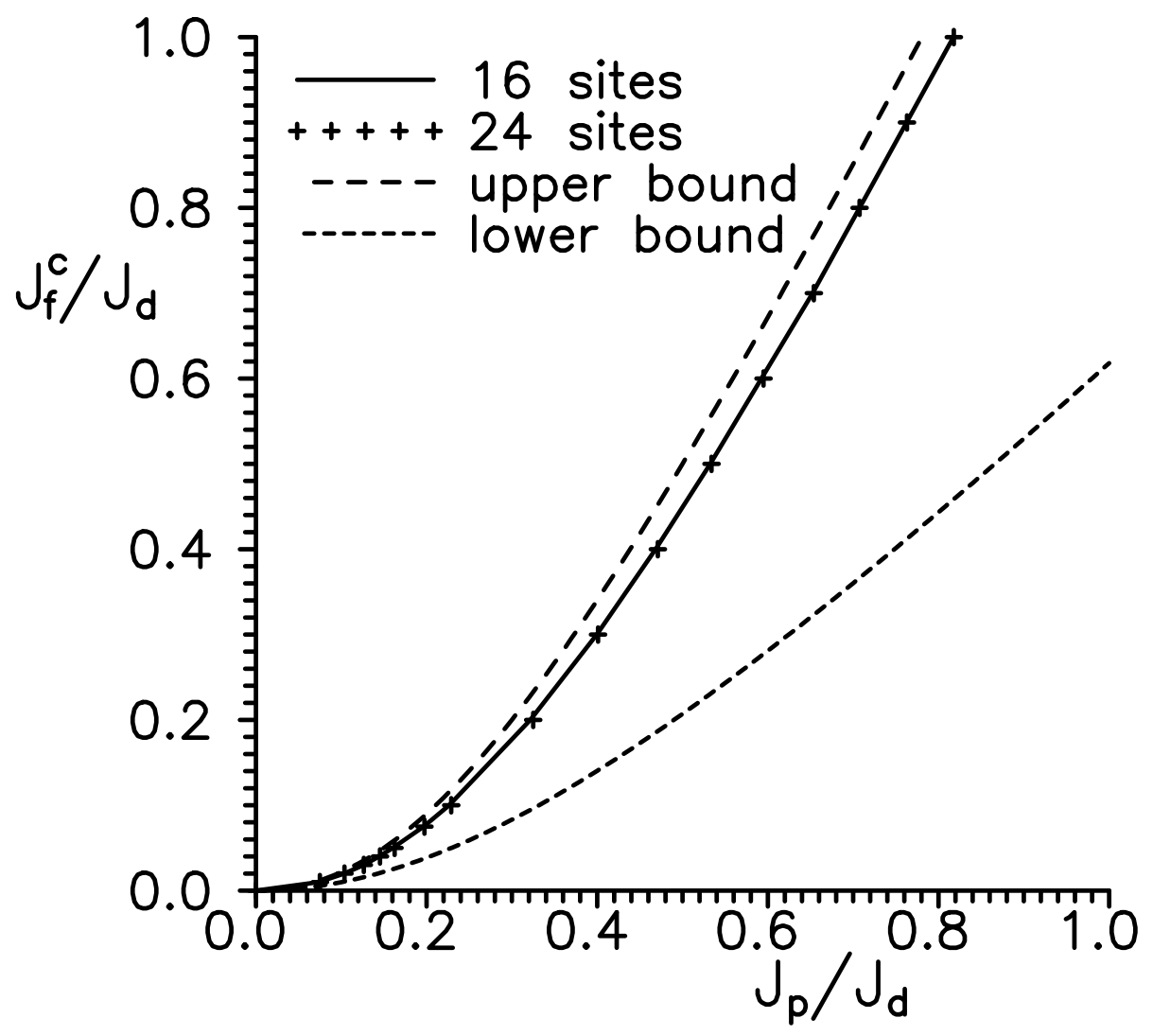

Fig.6 J.Richter et. al. 


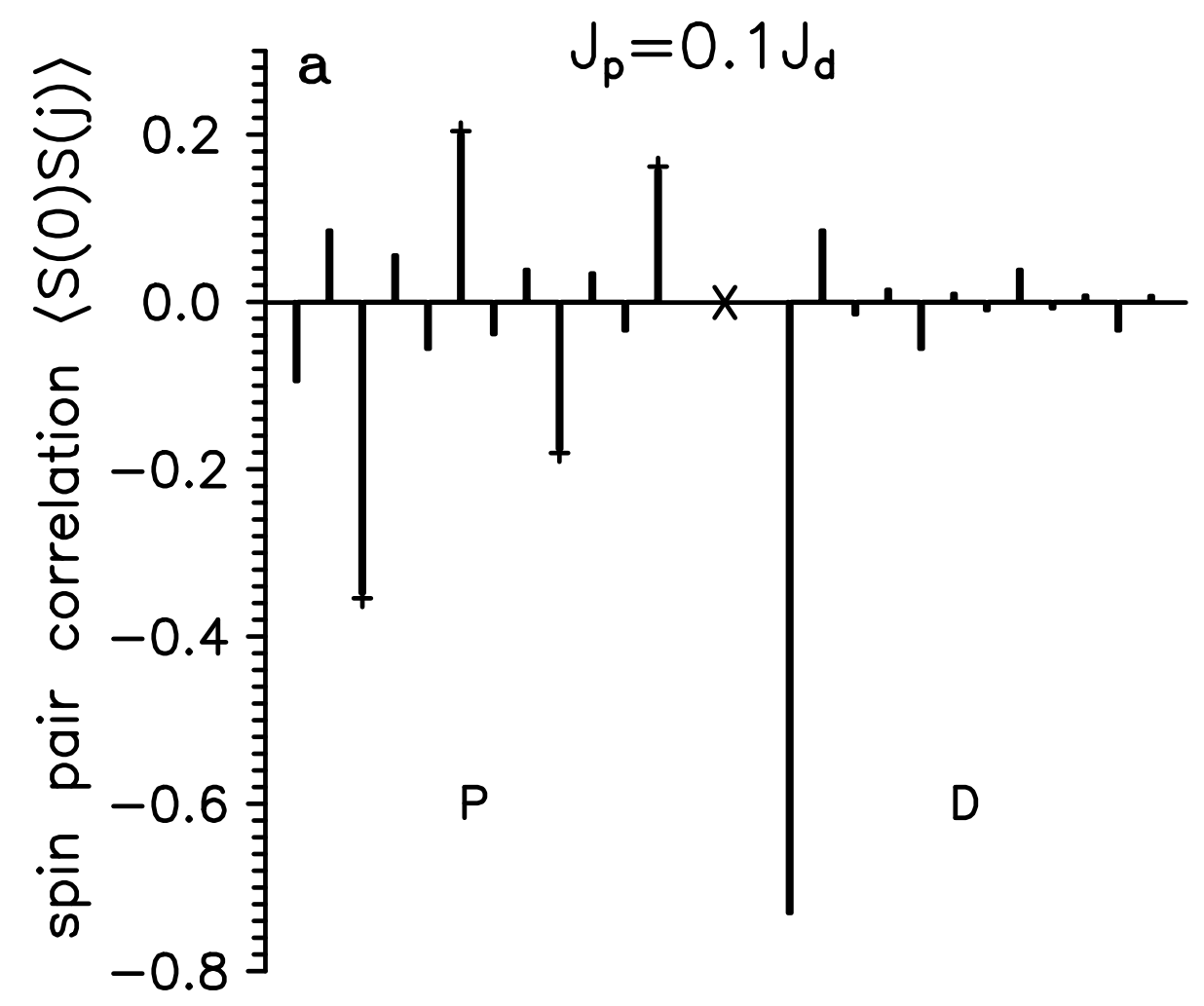

Fig.7a J.Richter et. al. 


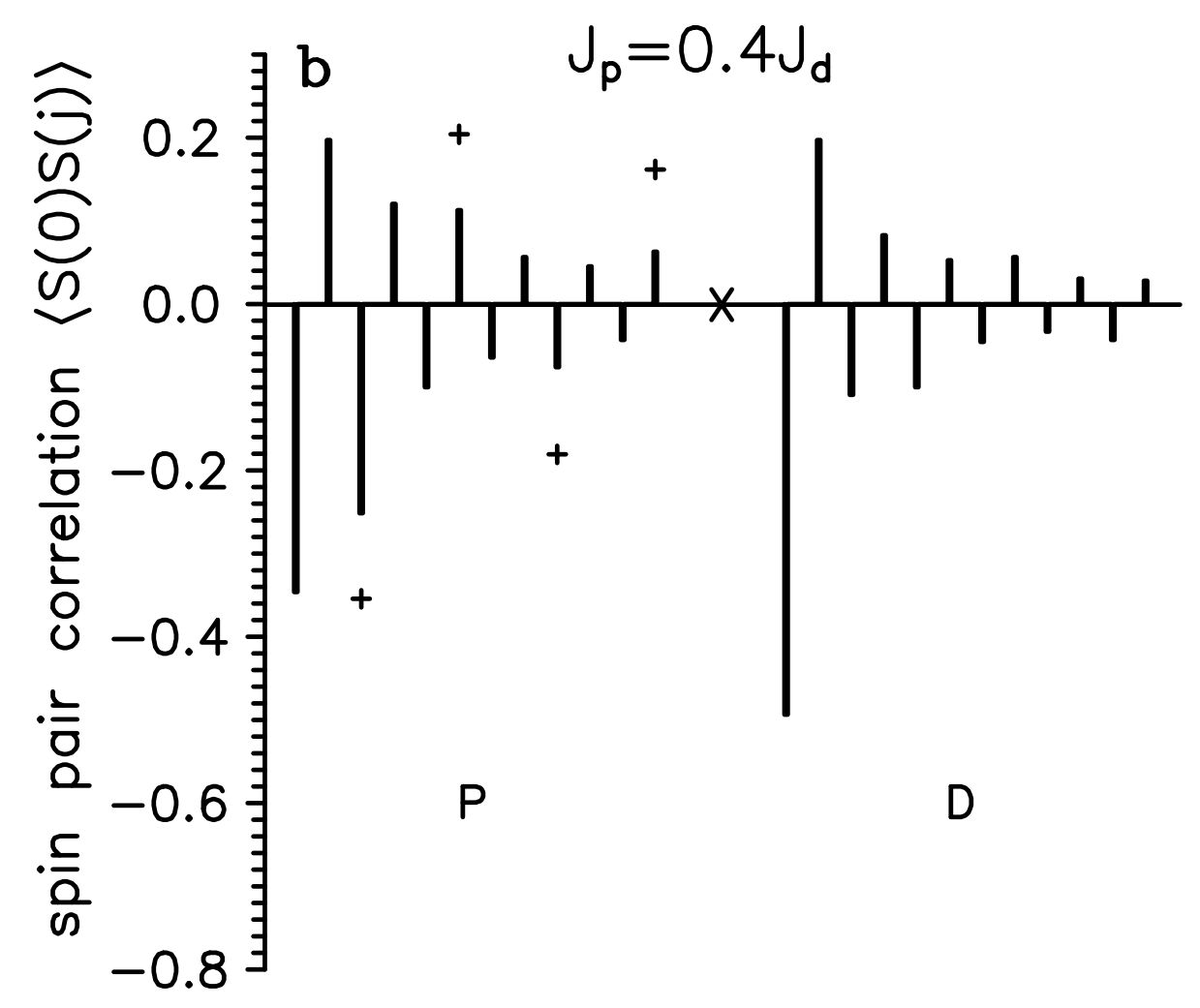

Fig.7b J.Richter et. al. 


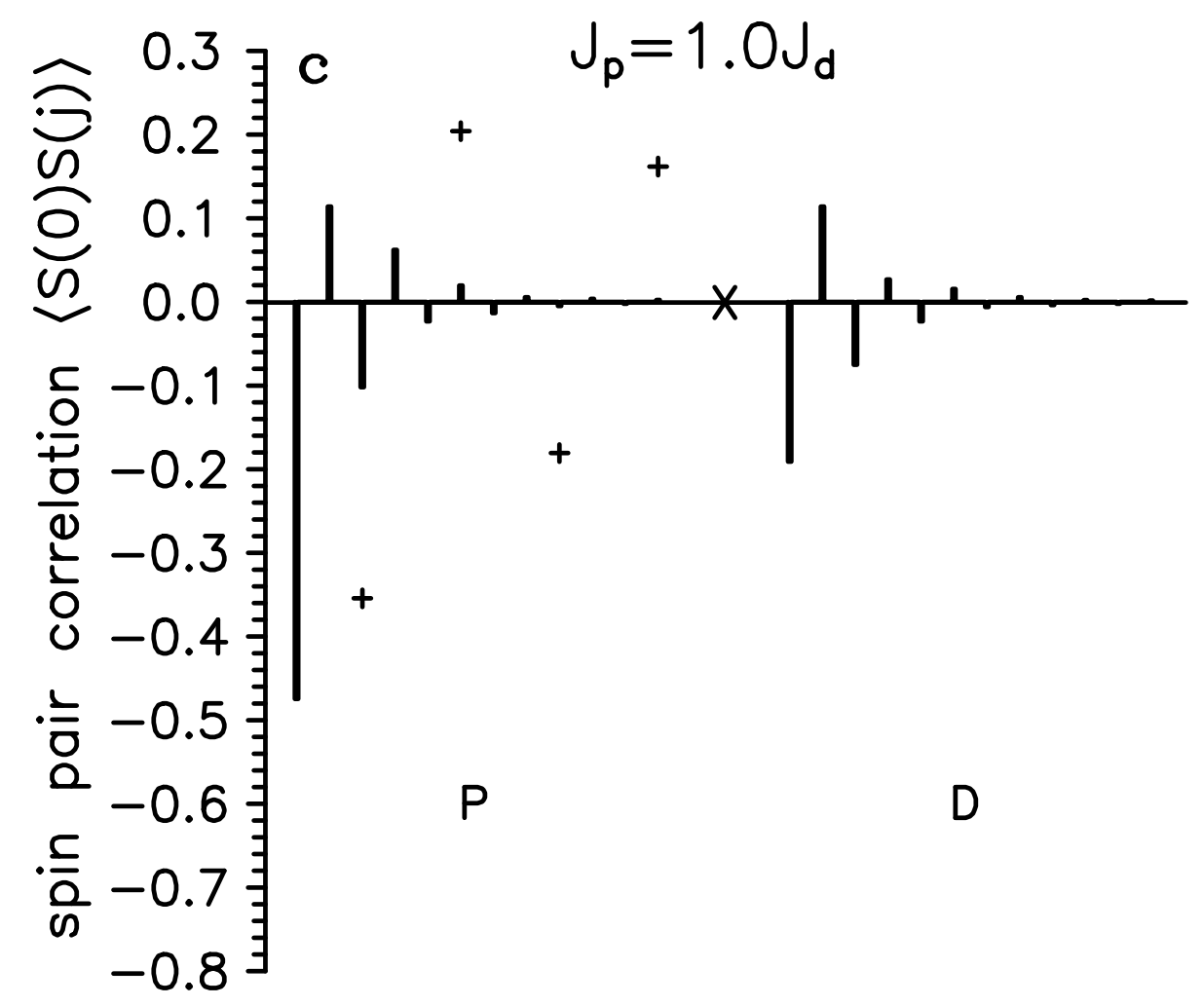

Fig.7c J.Richter et. al. 


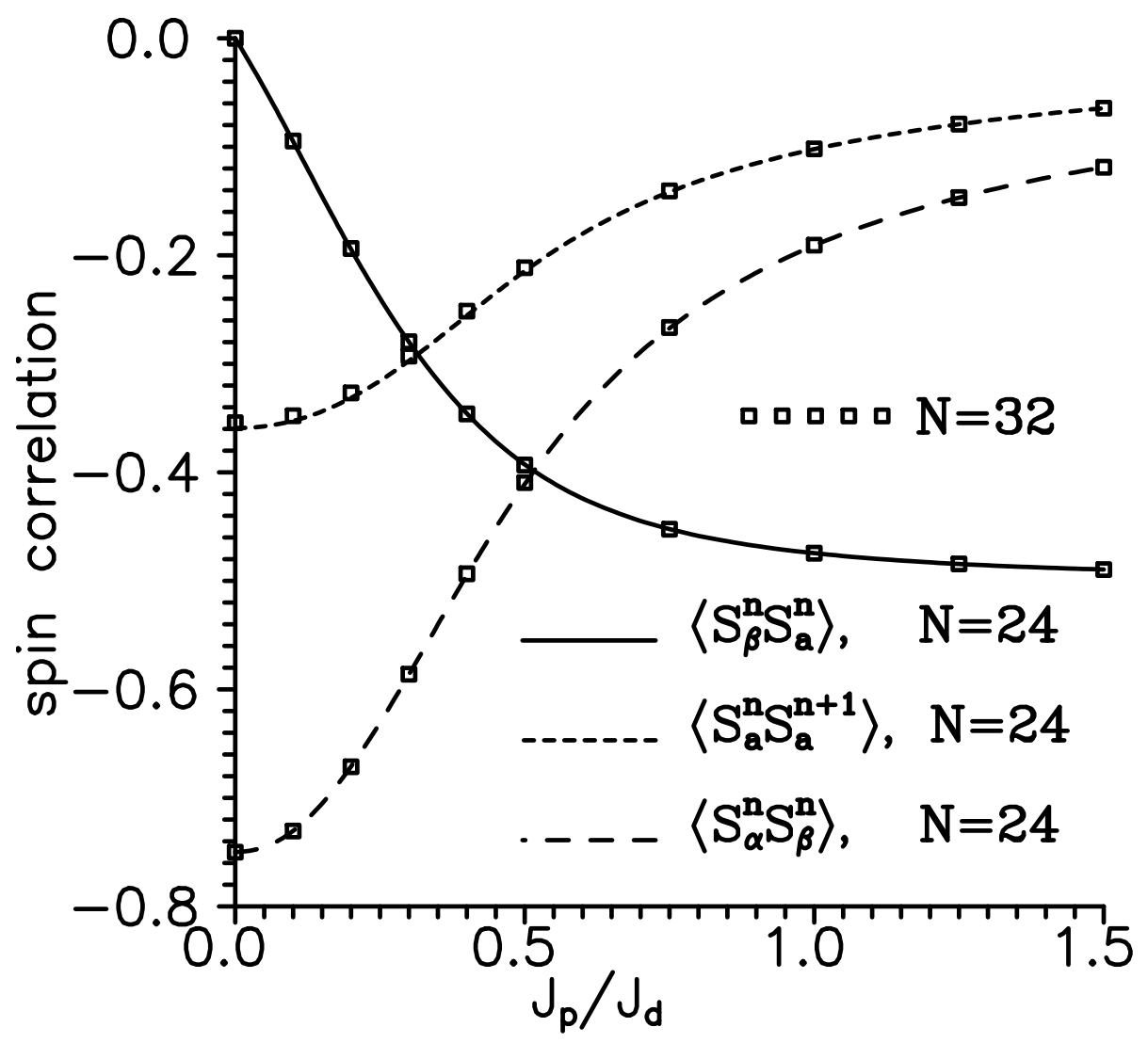

Fig.8 J.Richter et. al. 


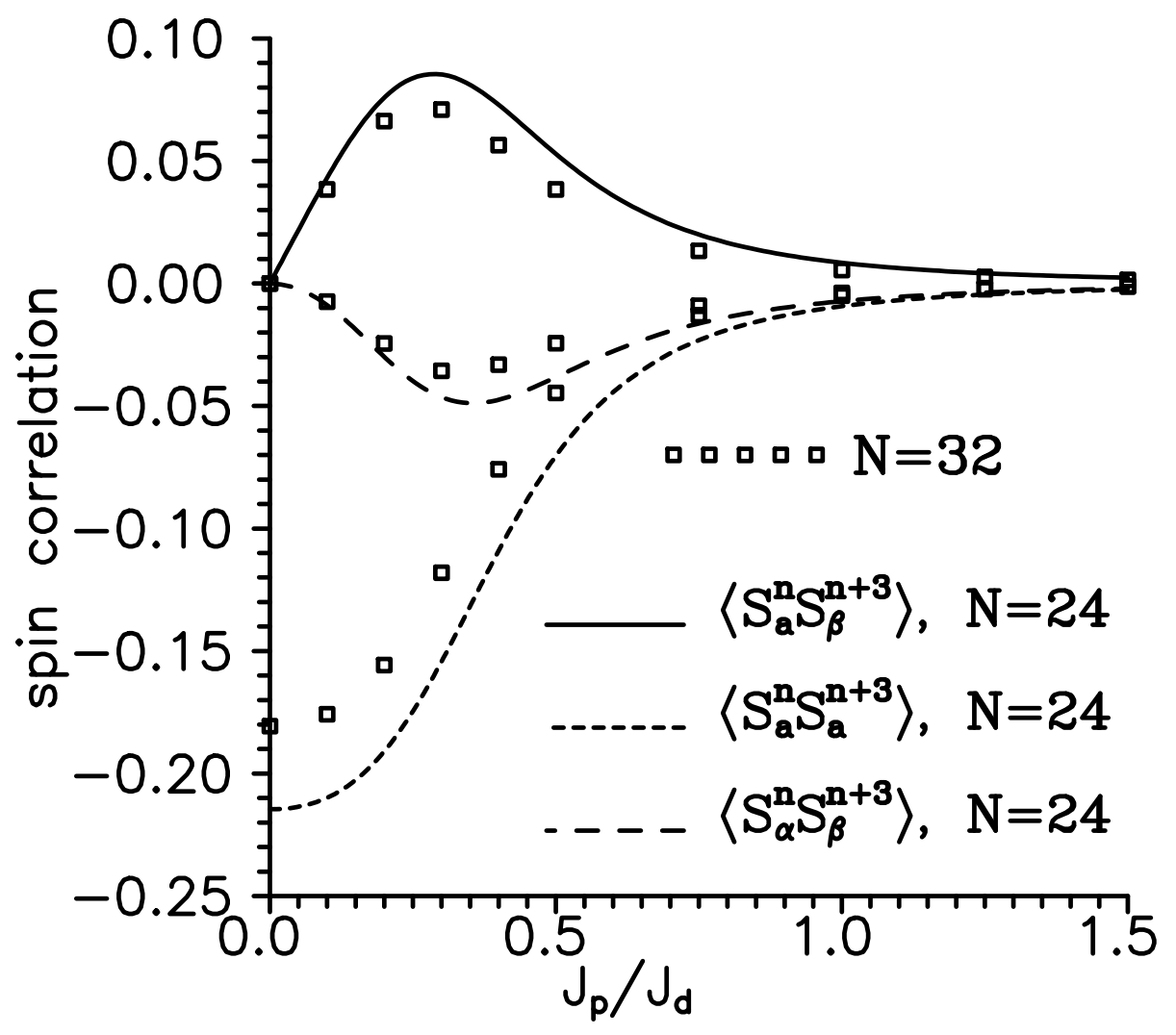

Fig.9 J.Richter et. al. 


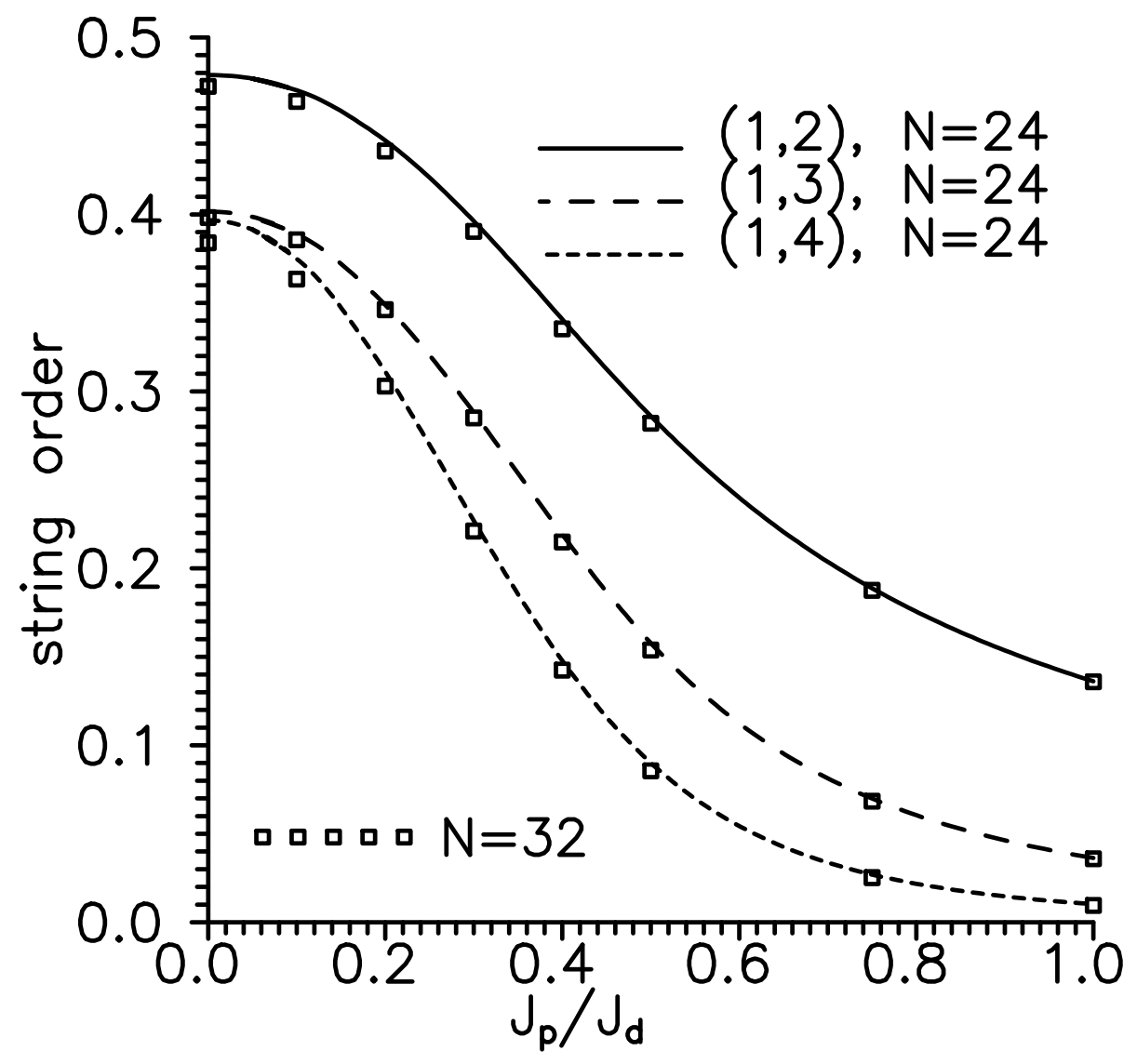

Fig.10 J.Richter et. al. 